\title{
Redes sociales \\ en proyectos ecoturísticos
}

DOI: 10.22403/UQROOMX/TYP07/05

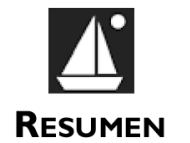

Martha Cecilia López Peredo

Carlos Arturo Torres Gastelú

Universidad Veracruzana

En las últimas décadas han cobrado importancia los procesos de aprendizaje que propician la innovación y son resultado de la configuración de redes como forma de organización social.

Los territorios son una construcción social en la que se integran aspectos sociales, culturales, económicos, ambientales, y que dan lugar a la conformación de redes locales de cooperación, formales e informales, las cuales propician la realización de proyectos de diferente índole e impulsan la innovación.

Este documento muestra los resultados sobre la generación de un proyecto económico para conformar una microempresa rural de ecoturismo, producto de la participación del sector público, de organismos no gubernamentales y participantes de la localidad. Se evidencia cómo los participantes se integraron en redes sociales, con familiares, amigos e instituciones públicas, para diversificar sus actividades económicas y crear una organización en la que se desarrollaron procesos de aprendizaje que modificaron sus actitudes, habilidades y valores, en beneficio de la comunidad y de las personas involucradas.

Palabras | Ecoturismo, microempresa, proyectos públicos, redes sociales. CLAVE 
Redes sociales en proyectos

ecoturísticos

\section{Introducción}

En los últimos años se ha destacado la importancia del conocimiento y de los procesos de aprendizaje como principal recurso específico de los territorios, de la innovación, en cuanto estrategia de respuesta frente a los retos de la globalización, o de la creación de redes como la forma de organización más adecuada para lograrlo. Así, un esfuerzo de innovación sostenido, entendido como "la capacidad de generar e incorporar conocimientos para dar respuestas creativas a los problemas del presente" (Méndez, 2002), resulta hoy un factor clave para mejorar la competitividad de las empresas y favorecer el desarrollo en los territorios.

En el ámbito de la economía espacial aparece otra perspectiva, rápidamente incorporada a una creciente cantidad de estudios geográficos, que sostiene que la innovación en las empresas es, en gran medida, resultado de la existencia de un entorno territorial (social, económico, cultural, etc.) con características específicas. Sin ninguna pretensión de dar origen a una nueva forma de determinismo ambiental, como en ocasiones han señalado sus detractores, los cada vez más numerosos estudios que desde comienzos de los años ochenta se han incorporado a esta visión parten de un hecho evidente: la concentración espacial que registran, por lo general, las empresas innovadoras.

A partir de esta realidad, la atención prioritaria se dirige a analizar e intentar comprender el ambiente en que nacen y operan esas empresas, procurando detectar la posible existencia de claves externas a la empresa pero internas a los lugares donde se dan los procesos de innovación. Los enfoques teóricos actuales encuentran una explicación a este hecho en la teoría de las ventajas competitivas de naciones y regiones, o en la del medio innovador $y$ las redes de innovación (Maillat y Grosjean, 1999; Maillat y Kebir, 1998).

Debe considerarse que los territorios son una construcción social, reflejo de acciones y comportamientos múltiples, acumulados en el tiempo, con capacidad de influir también de forma significativa sobre la estructura y el funcionamiento de las empresas. En ellos se lleva a cabo un proceso de desarrollo que, a partir de las aportaciones teóricas de las últimas décadas y dejando de lado el enfoque shumpeteriano, ahora es entendido desde una perspectiva integradora en la que se subrayan sus componentes sociales (bienestar), ambientales (sostenibilidad), políticos (gobernabilidad y participación local), culturales (defensa de la identidad y el patrimonio) y geográficos (ordenación del territorio), 
con el fin de lograr mayores niveles de calidad de vida que disfrutan las sociedades; es decir, se está en la búsqueda de un desarrollo territorial integrado.

De acuerdo con Ricardo Méndez (2002), se puede hablar de un territorio innovador en el que se tienen en cuenta no sólo los aspectos económicoempresariales, sino también los geográficos, lo cual permite incluir elementos no económicos, como el clima social apto para incorporar nuevas modalidades de trabajo o considerar la existencia de redes locales de cooperación, formales o informales, que hagan posible la realización de proyectos comunes, ya sea con objetivos económicos o de otra índole (culturales, de apoyo social, etc.), y que en ocasiones pueden concertarse para impulsar de manera directa diferentes formas de innovación.

Otros factores a considerar son la presencia de instituciones públicas, locales y regionales, que adoptan una actitud protagonista en el apoyo a la innovación y el desarrollo territorial mediante la generación de iniciativas propias; la negociación de acuerdos con instancias públicas o privadas, que aseguren una suficiente participación de la sociedad civil en los procesos de información y decisión; así como un esfuerzo por mejorar la formación de los recursos humanos, que puede implicar la enseñanza reglada en sus diversos niveles, la cualificación y reciclaje de empresarios y trabajadores, o una adecuada adaptación de la oferta formativa a las demandas y al saber hacer locales.

Los procesos de innovación en el seno de las empresas y de los sistemas productivos exigen trascender estos aspectos de investigación para fijarse en la posible existencia de redes sociales de apoyo.

El cambio en la unidad de estudio, que pasa de la empresa al territorio, obligó a considerar las complejas redes de relaciones que lo organizan. En ese sentido, desde los enfoques relativos al contexto institucional de las empresas, local embeddedness (Granoveter, 1985) o encastrement (Grossetti, 200I), hasta la perspectiva de la economía solidaria (Mance, 2000), han hecho interesantes aportaciones. En palabras de Sforzi (1999),

el éxito de una empresa ya no se relaciona sólo con el dinamismo del sector al que pertenece y con una superior capacidad interna de inventar nuevas soluciones [sino que], al lado de las relaciones económicas de producción, asumen un papel esencial las relaciones sociales de cooperación y la compenetración de las empresas en las redes de relaciones socioeconómicas que constituyen el sistema local, entendido como integrador versátil de conocimientos y organización (cit. por Méndez, 200I). 
Redes sociales en proyectos

ecoturísticos

En este caso se requiere que organismos públicos y privados cooperen con las empresas locales o los nativos en el desarrollo de proyectos comunes de carácter innovador, y que prevalezca un clima de confianza que propicie la participación de los actores locales, ya que la ausencia de redes locales de colaboración se convierte en un obstáculo para la innovación y el desarrollo.

Este enfoque de territorio innovador no sólo se aplica en el entorno de las grandes o medianas empresas, sino que, consideramos, tiene un enfoque holístico y se centra en todo tipo de unidades de producción, independientemente de su giro o su tamaño.

En el mundo actual, $90 \%$ de la producción de bienes y servicios proviene de pequeñas empresas o Pymes, situación que pone de manifiesto la relevancia económica que ellas representan para la economía en general. Este tipo de organizaciones se localizan en cualquier parte, llámese distrito industrial, cluster, ciudad, pueblo, localidad, calle, etc., y se encuentran desempeñando una importante actividad ya sea de apoyo administrativo, de producción o de distribución, entre otras.

Las microempresas -sector económico que, como una alternativa creativa, una persona, familia o grupo de personas genera- se ubican tanto en el contexto urbano como en el rural. En este trabajo se profundizará en las del ámbito rural y se hallará que ahí, para avanzar, las microempresas se apoyan en la conformación de redes sociales, lo cual permite enfrentar las principales restricciones derivadas del escaso desarrollo de los mercados y de los servicios públicos en el entorno rural.

La integración de microempresas rurales es una estrategia de sobrevivencia de los habitantes de estas zonas y de mejoramiento de su calidad de vida. Utilizan las redes sociales que las proveen de información necesaria para su implementación, operación y desarrollo. En muchos lugares, las microempresas han sido resultado de un proceso de reconversión productiva. Por ejemplo, en aquellos sitios tradicionalmente agrícolas o ganaderos, al disminuir la calidad de las tierras o el volumen de producción, se requiere buscar alternativas de autoempleo, que por lo general encuentran en microempresas de servicios o comerciales.

El concepto de redes sociales alude al conjunto de personas unidas por lazos de parentesco, sin que ello implique necesariamente una unidad de convivencia, en cuyo seno se establece un sistema de relaciones e intercambios de intensidades diferenciadas en los distintos segmentos del conjunto (Montes 
Urday y Catacora Bruna, 2005). De ahí que las redes sociales se conviertan en un importante instrumento que permite el análisis de nuevos roles y estructuras familiares, entre los cuales caben destacar las relaciones de confianza, reciprocidad y cooperación en su interior -entre los diferentes miembros de la familia-, $y$ en el exterior, con otros grupos y comunidades.

En la conformación de las redes sociales participan actores internos que, como ya se mencionó, son los familiares y personas más cercanas al grupo; y externos, como las dependencias del sector público, asociaciones civiles o no gubernamentales interesadas en impulsar el desarrollo local de comunidades rurales que necesitan algún tipo de apoyo en materia de organización, intermediación financiera, aspectos técnicos, de capacitación, etcétera.

\section{Objeto de estudio}

En el estado de Veracruz existe una gama de posibilidades de desarrollo local a partir de la innovación y las redes sociales. Nos centraremos en una zona geográfica al sur de la entidad, ubicada en la región de Los Tuxtlas, lugar de gran riqueza biótica, hidrológica, arqueológica, cultural y escénica, que busca la protección y conservación de la biodiversidad mediante la participación de los grupos locales. Para ello, se torna imperiosa la colaboración de organismos públicos que promuevan una política de sustentabilidad con un enfoque social y de autogestión.

Esta región, en términos de política pública, es un área natural protegida (ANP), que se oficializa como tal en 1998 por medio de un decreto publicado en el Diario Oficial de la Federación, conforme al cual se constituye la Reserva de la Biosfera de Los Tuxtlas. En esta iniciativa, los objetivos de conservación se conjugan con la obtención de beneficios económicos y oportunidades para mejorar las condiciones de vida de la población directamente involucrada con la propiedad y uso de los recursos naturales (Unesco, 197I).

En este modelo de desarrollo se asume que el mejoramiento de la calidad de vida, la reorientación de los esquemas productivos y la disminución de la presión sobre los recursos, hacen que el involucramiento de la población sea importante, así, se convierte en un modelo participativo que incorpora a los locales y a otras instituciones como gestores del desarrollo territorial integrado. 
Redes sociales en proyectos

ecoturísticos

Los órganos públicos que participan, y que son considerados por la Secretaría de Medio Ambiente y Recursos Naturales (Semarnat), son la Comisión Nacional de Áreas Naturales Protegidas (Conanp) y el Consejo de Asesores, además de instituciones académicas, centros de investigación y organizaciones no gubernamentales, entre otros. Los programas operativos son el Programa de Desarrollo Regional Sustentable (Proders), Manejo Integrado de Ecosistemas (MIE) y Global Environment Facility (GEF), cuyos objetivos generales son combatir la pobreza, mejorar el bienestar social y detener los procesos de degradación ambiental. Para lograrlo, las estrategias que implementan están dirigidas a proporcionar asistencia técnica que les permita diseñar estrategias participativas, mediar en el diálogo entre los actores involucrados y construir local y regionalmente las capacidades institucionales para instrumentar el manejo participativo, con lo que se convierten en promotores de la innovación y la construcción de redes sociales.

Desde 1997, las acciones derivadas del Proders se han enfocado en las siguientes áreas: ecoturismo, conservación de suelos, plantaciones forestales, artesanía, ecotecnias, acuacultura e intensificación de la ganadería extensiva hacia modelos silvopastoriles. Para poner en operación estas acciones, el personal de la reserva imparte diferentes cursos de capacitación a los integrantes de la comunidad que estén interesados en colaborar en alguno de los proyectos que se van a financiar.

Las comunidades atendidas por estos programas son elegidas de conformidad con los siguientes criterios (Paré y Tajín, 2007):

a) Ubicación geográfica en el límite con algunas de las tres zonas núcleo

b) Que hayan tenido previamente iniciativas hacia la reconversión productiva (ecoturismo, café de sombra, ixtle)

c) Que sus actividades productivo-extractivas no sean sustentables (ganadería, milpa, tala, extracción de fauna y no maderables)

d) Que hayan sido afectados por la expropiación

e) Que sean de alta marginación

Las acciones de las dependencias se dan en el ámbito comunitario y buscan cambios concretos en la gestión comunitaria y en el manejo sustentable de recursos naturales. 
Con base en estas consideraciones, las acciones que realiza la Reserva de la Biosfera de Los Tuxtlas se resumen en promover la protección y restauración de los recursos naturales y en mitigar la pobreza extrema en que viven las comunidades de la región, a través de la conversión de las actividades productivas y extractivas hacia modelos que permitan mantener vigentes los valores naturales de la región.

De los proyectos de reconversión productiva impulsados y apoyados por este organismo surge en el año 2000 la microempresa rural de ecoturismo Los Manglares de Sontecomapan.

Sontecomapan, localidad del municipio de Catemaco, Veracruz, es una gran laguna costera formada por la desembocadura, en el Golfo de México, de los ríos que bajan de la sierra. La unión del agua dulce (ríos) y salada (mar) ha creado el ambiente adecuado para que abunde el mangle, árbol de enormes raíces en las que proliferan los cangrejos rojos y azules, los cuales se observan durante el paseo en lancha, a la vez que se miran numerosas aves acuáticas y otros animales. El manglar de Sontecomapan es considerado uno de los mejor conservados y menos contaminados del país y cuenta con una extensión aproximada de 89I hectáreas (Contreras y Castañeda, 1995).

La población de la localidad de Sontecomapan es eminentemente rural (2 500 habitantes) con características propias de este tipo de lugares. Las actividades económicas predominantes son la agricultura, la ganadería, la pesca y los servicios relacionados con el turismo.

\section{Formación de redes en una microempresa rural de ecoturismo}

La microempresa Grupo Ecoturístico Los Manglares de Sontecomapan surge a partir de una invitación por parte de la Reserva de la Biosfera de Los Tuxtlas en el año 2000. Al respecto, Ofelia (2006)' señala: "y se viene una serie de talleres, y no te hablan de un proyecto, te hablan de la importancia de los manantiales, nuestra laguna, los ríos, acerca de cómo la tala está siendo tan tremenda y cómo se está desbastando..." Al realizar la invitación en esos talleres, el organismo público impulsa la reconversión productiva de la zona, pero

' Miembro de la comunidad ecoturística de Sontecomapan; sus principales funciones son promoción y ventas, cocinera y artesana; entrevistas realizadas en agosto, octubre y diciembre de 2006 y en febrero y abril de 2007. 
Redes sociales en proyectos

ecoturísticos

ante todo busca concienciar a los habitantes de la localidad acerca de la importancia de su riqueza natural, para que su explotación promueva la conservación del territorio.

Los miembros de la microempresa son pobladores de la localidad, y se constituyeron a partir de las redes locales actuales, con lo cual se ha fortalecido la continuidad de la comunidad ecoturística -por los lazos existentes y aquellos que se van configurando.

Las redes sociales se tejen a partir de las relaciones familiares, $y$, en su integración, los participantes tienen que romper obstáculos sociales y culturales que predominan en la comunidad:

se forma un grupo de 13 personas, y esas 13 personas pasan los talleres, porque incluso yo llego a formar parte del grupo cuando ya está la lista de la gente [...] la invitación de participar me la hace mi hermano, él no forma parte del grupo porque emigró a los Estados Unidos, entonces él me dice que formara parte del grupo porque se van a cuidar parte de los ríos y le hablan de un apoyo al esfuerzo que le vas a dar, entonces en esa parte yo digo será bueno, Ángel mi esposo ya andaba y resulta que no quería que perteneciera al grupo, pues él no quiere porque, como siempre, el machismo predomina en la comunidad... [Ofelia, 2006].

La Reserva de la Biosfera estableció como requisito la participación tanto de hombres como de mujeres de la comunidad, por lo que ésta se vio forzada a aceptar tal situación. Las actividades típicas de los hombres son como campesinos, albañiles o pescadores, mientras que las mujeres se dedican al cuidado de la familia; aunque también una a la elaboración y venta de pasteles, y otra a la manufactura y venta de artesanías. Por tanto, la actividad ecoturística se convierte en un complemento de la economía familiar.

El financiamiento concedido a la microempresa ha sido público, por parte del Proders, el Fondo Mexicano, A.C. y Conanp. El financiamiento ha sido a fondo perdido y los beneficiados han tenido que dar faena gratis, lo cual, de acuerdo con los testimonios de la responsable de los proyectos ecoturísticos de la Reserva de la Biosfera, compensa casi toda la inversión. ${ }^{2}$ Para todos los financiamientos, el aval y la supervisión del uso de los recursos ha sido responsabilidad de la Reserva de la Biosfera.

La infraestructura de la microempresa se encuentra ubicada en un área de varias hectáreas a dos kilómetros de la localidad de Sontecomapan. Es

${ }^{2}$ Entrevista a Katia, encargada de la Dirección de la Reserva de la Biosfera, octubre de 2007.

TEORÍA Y PRAXIS

7 (2009: I0I-II4) 
propiedad ejidal,y el dueño es uno de los participantes en el grupo, esposo de Ofelia.Tiene una cabaña terminada, con capacidad para 24 personas y otra que está en construcción, la cual tendrá cabida para 16 personas (cuatro por recámara), ambas construidas con recursos públicos. Cuenta con dos baños, que están afuera de las cabañas, uno para hombres y otro para mujeres, y tienen la particularidad de ser baños secos, construidos con principios ecotécnicos. Las regaderas se encuentran en un área separada y tienen un depósito para el agua jabonosa. También hay un comedor y una cocina. Otras construcciones en proceso son un iguanario y un museo temático.

La estructura administrativa de la microempresa está formada por un presidente, un secretario, un tesorero y vocales, lo cual responde a las necesidades de la constitución legal del grupo, más no a las de una unidad productiva como tal, que carece de estructura.

La distribución de puestos y responsabilidades en la organización es de tipo aleatorio, ya que todos pueden desarrollar las mismas actividades en las diferentes áreas operativas: cocina, comedor, recámaras, baños, guías, etc., sin distinción de sexo o edad. Esto se da porque todos han recibido capacitación para desempeñar los servicios necesarios en el funcionamiento de la microempresa y en ofrecer atractivos turísticos en el marco de la sostenibilidad.

La capacitación en materia de promoción, impartición de talleres artesanales, guías de turistas, aspectos administrativos, costeo de alimentos, entre otros, ha estado financiada por la Secretaría de Turismo. También se han realizado intercambios de conocimientos por medio de viajes a otras comunidades fuera del estado de Veracruz. Por ejemplo, Bioplaneta, A.C. apoyó con los intercambios de ideas y experiencias en proyectos de ecoturismo:

así como nosotros, los intercambios se van dando y nosotros visitamos y habían de otros estados, por ejemplo Oaxaca, estuvimos en un recorrido por ocho días por toda la sierra de pueblos que están haciendo ecoturismo [...] nos dimos cuenta de que ellos ya estaban más formados y entonces [nos dijeron] así no le hagan porque ahí nosotros tropezamos, así sí tuvimos muchos errores [...] ahora se piensa más para hacer las cosas y no nada más así al aventón... [Ana Josefa, $2007]^{3}$

${ }^{3}$ Miembro de la comunidad ecoturística de Sontecomapan; sus principales funciones son promoción y ventas, cocinera y ex presidenta; entrevistas realizadas en agosto, octubre y diciembre de 2006 y febrero y abril de 2007. 
Redes sociales en proyectos

ecoturísticos

La capacitación de instituciones y las visitas a otros lugares han permitido a los integrantes de la microempresa generar un conocimiento vivencial y local de las actividades que realizan. La transmisión de conocimientos se ha dado por la participación en redes sociales de apoyo.

La comercialización de sus servicios la hacen participando en expo ferias, para lo cual "escogemos al mejor exponente para asistir a las expo ferias, entonces lo becamos..." (Juan, 2007). ${ }^{4}$ Entre todos absorben los viáticos. En estos eventos la microempresa se da a conocer por medio de folletos y explicando a los interesados el tipo de servicio que se ofrece, con lo que la red se sigue construyendo: "principalmente los contactos que hagas es muy importante, como por ejemplo por una cadena de televisión o la radio o gente que trabaja para diferentes comunidades o instituciones que te pueden apoyar, y lógico que vas y armas tu paquete para que la gente te visite..." (Ofelia, 2007).

En las actividades regionales o nacionales que participan promueven sus servicios, pero también son áreas de oportunidad, pues los contactos que establecen les permiten tener otras fuentes de financiamiento para nuevos proyectos. Su mercado es sólo regional y nacional (no tienen presencia en el estado ni en la localidad).

Aparte del alojamiento y las comidas, ofrecen temascal, artesanías, sendero interpretativo, sendero acuático, la Cueva de los Murciélagos y la Poza de los Enanos. Para brindar estos servicios, todos los integrantes se han capacitado y han aprendido estas temáticas, utilizando los materiales de la región; por ejemplo, en las artesanías, aprendieron a tejer el lirio acuático, con el que elaboran bolsas y tapetes, entre otras cosas; mientras que con las semillas de frutos y granos que se cultivan en la región producen aretes y collares. Entre los participantes de diferentes comunidades se va dando la transmisión de conocimientos y de técnicas de elaboración.

Esta microempresa no ha escapado de las dificultades: ha afrontado problemas de organización, de control, de financiamiento, de comercialización, de publicidad, etc., los cuales se han resuelto en mayor o menor medida con la participación de sus integrantes, que han buscado apoyo en las instituciones

\footnotetext{
${ }^{4}$ Miembro de la comunidad ecoturística de Sontecomapan; sus principales funciones son guía, mantenimiento de las instalaciones y entorno natural, miembro de vigilancia de la Procuraduría Federal de Protección al Ambiente (Profepa) y presidente actual; entrevistas realizadas en agosto, octubre y diciembre de 2006, y febrero y abril de 2007.
} 
oficiales que trabajan con ellos, como la Reserva de la Biosfera, algunas organizaciones no gubernamentales, asociaciones civiles y universidades.

En el aspecto administrativo, han enfrentado problemas de liderazgo,ya que, al darse el cambio en la presidencia de la directiva, algunos miembros no estaban de acuerdo con la forma en que se estaba manejando la microempresa y entraron en una lucha de poder. Esta situación afectó el funcionamiento de la organización y, para resolver los problemas, intervino la Dirección de la Reserva, enviándoles a un consultor que les dio un taller de manejo de conflictos.

Al inicio de las actividades, los integrantes de la organización recibieron un taller sobre cómo debían administrar la microempresa y llevar su contabilidad. En cuanto al problema de financiamiento, lo han resuelto de dos formas: con recursos internos y externos. En el primer caso, por ejemplo, al realizar el balance de los ingresos obtenidos en una temporada de verano -que es cuando reciben más turistas- $y$, una vez cubiertos sus sueldos, reinvirtieron las utilidades en la compra de una celda solar, de artículos para la cocina (platos, cacerolas, vasos, etc.), blancos para las cabañas, entre otras cosas. Respecto a los recursos externos, el financiamiento lo obtienen al participar en proyectos de instituciones federales, internacionales, o de organizaciones no gubernamentales.

En la comercialización, su principal problema ha sido la publicidad, el cual se ha solventado en parte con el apoyo de las instituciones de educación superior que realizan actividades de investigación y colaboración en esta región. En principio, la Universidad Autónoma Metropolitana les diseñó un tríptico con el que daban a conocer sus servicios cuando asistían a ferias y a las expo, sobre todo en la Ciudad de México.

En 2006, la Universidad Veracruzana, por medio de los integrantes del Cuerpo Académico (CA) Tecnologías de la Información y estudiantes de servicio social, les diseñaron un curso de manejo de conflictos; un tríptico, en el que se actualiza la imagen de la empresa -pues se bosqueja un logotipo y una planeación estratégica-; y una página web utilizando software libre.

Todo lo anterior permite concluir que los integrantes de la microempresa han empleado redes de apoyo social en la resolución de sus conflictos, recurriendo a instituciones del sector público para tal fin; es decir, se han valido de la transmisión del conocimiento y de los procesos de aprendizaje, como principal recurso específico de los territorios, de la innovación como estrategia de respuesta frente a los retos de la globalización, o de la creación de redes como 
Redes sociales en proyectos

ecoturísticos

la forma de organización más adecuada para lograrlo, respetando siempre el entorno territorial (social, económico, cultural, etc.) con características concretas definidas a priori.

Esto implicó un proceso de análisis y de comprensión del medio ambiente en el que nació y en el que opera la microempresa, intentando detectar algunas claves externas a ella -organismos del sector público- pero internas respecto a los lugares donde se dan los procesos de innovación. En la existencia de recursos naturales es donde se originan las ventajas competitivas de la organización, la cual, con su trabajo y con la conservación de los recursos naturales, está innovando en la oferta de un servicio que le representa una actividad económica alternativa, de la cual sus miembros obtienen recursos económicos complementarios a sus ingresos de agricultores, ganaderos, comerciantes, entre otros.

\section{Conclusiones}

Todos formamos parte de redes, no habría otra posibilidad, es la ley de la vida social. No son por completo evidentes, pero actúan cuando hace falta, y entonces nos sorprenden. Otras veces son movilizadas a voluntad, se sabe de su existencia, y a propósito las ponemos en acción. Somos parte de redes, pero no somos expertos en su formación ni en su desarrollo (Galindo, 2006: 62).

Cuando aparecen tienen otros nombres: familia, amigos, compañeros de trabajo. Actúan según la calidad de los vínculos que hemos formado. Si hemos invertido energía en ellas, nos cubren con su extensión cuando hace falta; si hemos faltado a su fortalecimiento, nunca se hacen presentes. $Y$ a veces, por efectos de configuración social, aun en nuestra ausencia, nos reconocen y apoyan.

Las redes no son instrumentos, pero funcionan como tales, se configuran en las circunstancias concretas. Las redes no son corporaciones garantizadas, pero toman esa forma y ayudan en situaciones particulares. Las redes actúan con la lógica de pares, nada hay que esperar de ellas, sólo se es parte,y de pronto operan como un cuerpo unificado y sentimos su poder.

El caso de la microempresa en estudio presenta evidencias de la creación de redes sociales a partir de la construcción de nichos ecológicos impulsados por políticas públicas y organismos externos que habilitan la formación de competencias en los individuos y que promueven el fortalecimiento de las redes que van estableciéndose. Esto sucede a partir de las interacciones que 
tienen los individuos que integran esta comunidad ecoturística, caracterizada por un complejo proceso de ajuste en las formas de trabajo, en las normas y los valores de convivencia y en la búsqueda de su supervivencia.

Esta microempresa es el reflejo de que el contacto con otras redes y configuraciones produce cambios en las habilidades, conocimientos y actitudes de sus miembros. Desde su formación, la microempresa se ha distinguido porque, entre más contacto hay con otros organismos, surgen más redes, producto de la interacción natural, redituando beneficios palpables. Es un hecho que, al incrementar sus redes, se aceleraron los procesos de cambio vinculados con los esfuerzos de innovación promovidos por actores externos -como la Reserva de la Biosfera- con la bandera de la protección al medio ambiente.

Por último, el caso en estudio demuestra que las relaciones de colaboración adquieren especial importancia cuando operan en el nivel local, logrando que la cercanía geográfica de los actores de las redes que brindan apoyo económico, asesoría, capacitación o supervisión sean determinantes en los procesos de desarrollo local y en los proyectos de innovación.

\section{FUENTES CONSULTADAS}

Casas, Rosalba (coord.) (200I). La formación de redes de conocimiento. Una perspectiva regional desde México. Barcelona:Anthropos.

Conanp (2005). Disfrutando de los Tuxtlas. Reporte anual de la Comisión Nacional de Áreas Naturales Protegidas. México: Conanp (LOGR05, 5). (2006) Programa de Conservación y Manejo de la Reserva de la Biosfera Los Tuxtlas [en línea]. México: Comisión Nacional de Áreas Naturales Protegidas.Disponible en:http://www.cofemermir.gob.mx/uploadtests/ 9727.59.59.1.Tuxtlas.-\%20Programa20de\%Conservaci\%c3\%b3n\%20 y\%20manejo.pdf [2007, 21 de noviembre].

Contreras, E.F.y O. Castañeda L. (1995). Los ecosistemas costeros, Gobierno del Estado de Veracruz/SEDAP.

Galindo Cáceres, Jesús (2006)."La internet y sus redes sociales. Comunicología e ingeniería en comunicación social de un fenómeno aún emergente". Razón y Palabra, Primera Revista Electrónica en América Latina especializada en comunicación [en línea]. Disponible en: www.razony palabra.org.mx. 
Redes sociales en proyectos

ecoturísticos

Granoveter, M. (1985)."Economic action and social structure: the problem of embeddedness". American Journal of Sociology 9I (3), 48I-5I 0.

Grossetti, L. (200I). "Les effets de proximite spatial dans les relations entre organizations: une question d'encastrements". Espacios et societies I0I-102, 203-219

Mance, E.A. (2000). A revoluçao das redes. Petropolis: Editora Vozes.

Maillat, D. y N. Grosjean (1999). "Globalisation et systèmes territoriaux de production". Neuchâtel: Université de Neuchâtel (Working Paper IRER 9906a).

L. Kebir (1998)."'Learning region et systèmes territoriaux de production”. Neuchâtel: Université de Neuchâtel (Working Paper IRER 9802a).

Méndez, Ricardo (2002). “Innovación y desarrollo territorial: algunos debates teóricos recientes". EURE (Santiago), 28 (84) [en línea]. Disponible en: http://www.scielo.cl/scielo.php?pid=S0250-7 I 6 I 2002008400004 \&script=sci_arttext [2008, 20 de julio].

Montes Urday, Alipio y Juan Catacora Bruna (2005). "El papel de las redes sociales en la reproducción y desarrollo de la microempresa rural no agrícola: la experiencia de Arequipa y Moquegua" [en línea].Arequipa: Centro de Estudios para el Desarrollo Regional (Ceder). Disponible en: http://cies.org.pe/files/active/I/PBCI 304.pdf

Paré, Luisa y Tajín Fuentes (2007). Gobernanza ambiental y políticas públicas en Áreas Naturales Protegidas. Lecciones desde Los Tuxtlas. México: Instituto de Investigaciones Sociales, Universidad Nacional Autónoma de México (Cuadernos de Investigación).

Semarnap (Secretaría de Medio Ambiente, Recursos Naturales y Pesca) (1998). "Decreto de Reserva de la Biosfera, la región de Los Tuxtlas". Diario Oficial de la Federación, 23 de noviembre de 1998, 6-21.

Sforzi, F. (1999). “La teoría marshalliana para explicar el desarrollo local”, en F. Rodríguez (ed.), Manual de desarrollo local. Oviedo:Trea Ediciones.

Unesco (197I). MAB Biosphere Reserves Directory, Programa Hombre y la Biosfera. 